\title{
P03-1-34 Poster session
}

\section{Nicotine modulates depression-like behaviors via regulating nAChR/Akt signalings in stressed mice}

\author{
Mizuki Uchida ${ }^{1}$, Akimi Kuwana ${ }^{1}$, Rina Mizoguchi ${ }^{1}$, Norio Ozaki $^{2}$, Akira Yoshimi $^{1}$, Yukihiro Noda ${ }^{1}$ \\ ${ }^{I}$ Division of Clinical Sciences and Neuropsychopharmacology, Meijo University Faculty and Graduate School of \\ Pharmacy, Japan, ${ }^{2}$ Department of Psychiatry, Nagoya University Graduate School of Medicine, Japan
}

Background and purpose: The cigarette smoker rate of patients with psychiatric disorders, including mood disorders (depressive or bipolar disorders) and schizophrenia, is higher than general population. The smoking incentive is considered as self-medication for own psychoses, including nicotine dependence by stimulating nicotinic acetylcholine receptors (nAChRs) in the central nervous system. However, the effects of smoking on the neuropsychological functions and its molecular mechanisms are still unclear. In the present study, we investigated the effects of nicotine on the depressive behaviors and involvement of nicotinic signal transduction in stressed mice.

Methods: The stressed mice were established by forcing to swim for $15 \mathrm{~min}$ to stress (day 1$)$. Saline or (-)-nicotine (0.3 $\mathrm{mg} / \mathrm{kg}$ s.c.) was treated to the mice for 7 consecutive days from the next day of exposure to forced swimming stress. The forced swimming test (FST) and social interaction test (SIT) were performed $30 \mathrm{~min}$ after (-) nicotine treatment on day 8 in mice pretreated with saline and (-)-nicotine repeatedly. The rewarding effect of (-)-nicotine was assessed by the conditioned place preference (CPP) test. The protein expressions in the prefrontal cortex (PFC) and hippocampus (HIP) were analyzed by western blot immediately after SIT.

Results: Stressed mice showed immobility in the FST and social behavior impairment in the SIT, indicating depressivelike behaviors. Social behavior impairment, but not immobility was attenuated by repeated, but not acute treatment with (-)-nicotine. (-)-Nicotine at the dose of $0.3 \mathrm{mg} / \mathrm{kg}$ did not affect the CPP performance. In the PFC and HIP, there was no difference in the protein expressions of $\mathrm{nAChR}$ aplha7, aplha4, and beta2 subunits across all groups. However, the phosphorylated protein expression of Akt was decreased in the HIP of stressed mice showing social behavior impairment.

Conclusions: These results suggest that repeated treatment with (-)-nicotine is useful and effectively for stress-induced depressive-like behaviors at the dose without rewarding effect. The remission of (-)-nicotine for social behavior impairment may be mediated via regulating $\mathrm{nAChR/Akt} \mathrm{signalings} \mathrm{in} \mathrm{stressed} \mathrm{mice.}$ 